\title{
ON CERTAIN SEMIGROUPS OF TRANSFORMATIONS THAT PRESERVE A PARTITION
}

\author{
MOSAROF SARKAR AND SHUBH N. SINGH
}

\begin{abstract}
Let $X$ be a nonempty set, and let $\mathcal{T}_{X}$ be the full transformation semigroup on $X$. For a partition $\mathcal{P}=\left\{X_{i} \mid i \in I\right\}$ of $X$, we consider the semigroup $T(X, \mathcal{P})=\left\{f \in \mathcal{T}_{X} \mid \forall X_{i} \exists X_{j}, X_{i} f \subseteq X_{j}\right\}$, the subsemigroup $\Sigma(X, \mathcal{P})=\left\{f \in T(X, \mathcal{P}) \mid X f \cap X_{i} \neq \emptyset \forall X_{i}\right\}$, and the group of units $S(X, \mathcal{P})$ of $T(X, \mathcal{P})$. In this paper, we first characterize the elements of $\Sigma(X, \mathcal{P})$. For a permutation $f$ of finite $X$, we next observe whether there exists a nontrivial partition $\mathcal{P}$ of $X$ such that $f \in S(X, \mathcal{P})$. We then characterize and enumerate the idempotents in the semigroup $\Sigma(X, \mathcal{P})$ for arbitrary and finite $X$, respectively. We also characterize the elements of $S(X, \mathcal{P})$. For finite $X$, we finally calculate the cardinality of $T(X, \mathcal{P}), \Sigma(X, \mathcal{P})$, and $S(X, \mathcal{P})$.
\end{abstract}

\section{INTRODUCTION}

We assume that the reader is familiar with elementary concepts of combinatorics and semigroup theory. Throughout the paper, let $X$ denote a set with more than two elements, and let $\mathcal{P}$ denote a partition of $X$. The symbols $\mathcal{T}_{X}$ and $\mathcal{S}_{X}$ will be used to denote the full transformation semigroup and the symmetric group on $X$, respectively. For a subset $A \subseteq X$, we denote by $A f$ the image of $A$ under $f \in \mathcal{T}_{X}$. A map $f \in \mathcal{T}_{X}$ preserves a partition $\mathcal{P}$ if for every $X_{i} \in \mathcal{P}$, there exists $X_{j} \in \mathcal{P}$ such that $X_{i} f \subseteq X_{j}$.

The notion of semigroups of transformations that preserve a partition is one of the most trending topics of semigroup theory. For a partition $\mathcal{P}$ of a set $X$, Pei [17] introduced and studied the subsemigroup

$$
T(X, \mathcal{P})=\left\{f \in \mathcal{T}_{X} \mid \forall X_{i} \in \mathcal{P}, \exists X_{j} \in \mathcal{P}, \quad X_{i} f \subseteq X_{j}\right\}
$$

of $\mathcal{T}_{X}$. Moreover, Pei proved in [17, Theorem 2.8] that $T(X, \mathcal{P})$ is exactly the semigroup of all continuous selfmaps on $X$ endowed with the topology having $\mathcal{P}$ as a basis. Since then, $T(X, \mathcal{P})$ and its subsemigroups have received considerable attention and their several fascinating algebraic and combinatorial aspects have been investigated (see for example [1, 2, 3, 9, 10, 11, 13, 18, 19, 20, 25, 26). There have also been a number of interesting works on certain generalizations of the semigroup $T(X, \mathcal{P})$ (see for example [7, 8, 21, 22, 23]).

Pei [20] studied the regularity and Green's relations in the semigroup $T(X, \mathcal{P})$. When $\mathcal{P}$ is a uniform partition of finite $X$, Pei [19] gave an upper bound for the rank of $T(X, \mathcal{P})$. Later, Araújo et al. 3] calculated the rank of $T(X, \mathcal{P})$ and thus settled a conjecture on the rank of $T(X, \mathcal{P})$ posed by Pei in [19]. Araújo et al. [1] also

2010 Mathematics Subject Classification. 20M15; 20M20.

Key words and phrases. Transformation semigroups; Permutation groups; Partitions; Idempotents. 
calculated the rank of $T(X, \mathcal{P})$ for an arbitrary partition $\mathcal{P}$ of finite $X$. Dolinka et al. characterized as well as enumerated the idempotents of the semigroup $T(X, \mathcal{P})$ for finite set $X$ in $[9$ and [10] for the uniform and non-uniform cases, respectively. The cardinality of particular classes of subsemigroups of the semigroup $T(X, \mathcal{P})$ have also been calculated (see for example [12, 24]).

Pei [19] also considered the group of units $S(X, \mathcal{P})$ of the semigroup $T(X, \mathcal{P})$ and observed that $S(X, \mathcal{P})$ is exactly the subgroup of all homeomorphisms on $X$ endowed with the topology having $\mathcal{P}$ as a basis, and called it the homeomorphism group. For a uniform partition $\mathcal{P}$ of finite $X$, Pei [19] further deduced an upper bound for the rank of $S(X, \mathcal{P})$. Later, Araújo et al. 3] calculated the rank of $S(X, \mathcal{P})$ when $\mathcal{P}$ is a uniform partition of finite $X$. The homeomorphism group $S(X, \mathcal{P})$ is also studied by Araújo et al. in [1].

For a partition $\mathcal{P}=\left\{X_{i} \mid i \in I\right\}$ of a set $X$, let

$$
\Sigma(X, \mathcal{P})=\left\{f \in T(X, \mathcal{P}) \mid X f \cap X_{i} \neq \emptyset \forall X_{i} \in \mathcal{P}\right\} .
$$

It is clear that $\Sigma(X, \mathcal{P})$ is a subsemigroup of $T(X, \mathcal{P})$. When $\mathcal{P}$ is a uniform partition of finite $X$, Araújo et al. [3] calculated the rank of the semigroup $\Sigma(X, \mathcal{P})$. For a finite $X$, some interesting properties of $\Sigma(X, \mathcal{P})$ are also investigated in [1].

The rest of the paper is organized as follows. In Section 2, we recall necessary concepts from semigroup theory and combinatorics and introduce notation used within the paper. In Section 3, we characterize the elements of the semigroup $\Sigma(X, \mathcal{P})$. For a permutation $f$ of finite $X$, we observe whether there exists a nontrivial partition $\mathcal{P}$ of $X$ such that $f \in S(X, \mathcal{P})$ in Section 4. In Section 5, we characterize and enumerate the idempotents in the semigroup $\Sigma(X, \mathcal{P})$ for arbitrary and finite $X$, respectively. Moreover, we characterize the elements of $S(X, \mathcal{P})$. In Section 6 , we finally calculate the cardinality of $T(X, \mathcal{P}), \Sigma(X, \mathcal{P})$, and $S(X, \mathcal{P})$ when $X$ is a finite set.

\section{Preliminaries and Notation}

In this section, we introduce relevant notation and recall concepts from combinatorics and semigroup theory that are requisite to the paper. We refer the reader to the standard books [4, 16] for more detailed information from combinatorics and semigroup theory, respectively.

Unless stated otherwise, we will use capital letter to denote nonempty subset, calligraphic letter to denote collection of subsets, and small letter to denote setelement, map, or positive integer. The letter $I$ will be reserved for an arbitrary indexing set. The set of all positive integers is denoted by $\mathbb{N}$. We will always presume that $m$ and $n$ are positive integers. The symbol $I_{m}$ denote the subset $\{1, \ldots, m\}$. The number of elements of a set $A$ is denoted by $|A|$ and is called the cardinality or size of $A$. A set of cardinality $n$ is called an $n$-element set. We write $A \backslash B$ to denote the set of all elements $x \in A$ such that $x \notin B$. We denote by $[a]$ the equivalence class of an element $a$ of a set $A$ under an equivalence relation on $A$. We denote by $\left(\begin{array}{l}n \\ r\end{array}\right)$ the number of $r$-element subsets of an $n$-element set. We will use $A=\left\{n_{1} \cdot a_{1}, \ldots, n_{k} \cdot a_{k}\right\}$ to denote the multiset $A$ with $n_{i}$ copies of $a_{i}$ for each $i \in I_{k}$. 
Let $X$ be a nonempty set. A partition of $X$ is a collection of nonempty disjoint subsets of $X$, called blocks, whose union is $X$. A partition is called trivial if it has only singleton blocks or a single block. A partition is called uniform if all its blocks have the same size. An $m$-partition is a partition that has exactly $m$ blocks. For $m, k \in \mathbb{N}$ with $m \geq k$, an $(m, k)$-partition is an $m$-partition that has exactly $k$ different size blocks. It is well-known that any partition of $X$ induces naturally an equivalence relation on $X$, and vice versa (cf. [16, Proposition 1.4.6]).

The composition of maps will be denoted by juxtaposition. A selfmap on a set $A$ is a map from $A$ to $A$. Let $f, g \in \mathcal{T}_{X}$. For $x \in X$, we will use $x f$ to denote the image of $x$ under $f$, and compose maps from left to right: $x(f g)=(x f) g$. The symbols $\operatorname{dom}(f)$ and $\operatorname{codom}(f)$ will be used to denote respectively the domain and the codomain of $f$. The pre-image of a subset $B \subseteq X$ under $f$ is denoted by $B f^{-1}=\{x \in X \mid x f \in B\}$. If $A, B \subseteq X$ such that $A f \subseteq B$, then there is a map $g: A \rightarrow B$ defined by $x g=x f$ for all $x \in A$ and, in this case, we say that $g$ is induced by $f$. If $X=\{1, \ldots, n\}$, we will write $\mathcal{T}_{X}$ and $\mathcal{S}_{X}$ as $T_{n}$ and $S_{n}$, respectively.

A permutation $f$ of $I_{n}$ is called a cycle of length $m$ or an $m$-cycle, denoted by $\left(a_{1}, \ldots, a_{m}\right)$, if there exists a subset $\left\{a_{1}, \ldots, a_{m}\right\}$ of $I_{n}$ such that $a_{i} f=a_{i+1}$ for all $i=1, \ldots, m-1, a_{m} f=a_{1}$, and $a f=a$ for all $a \notin\left\{a_{1}, \ldots, a_{m}\right\}$. Any two cycles $\left(a_{1}, \ldots, a_{k}\right)$ and $\left(b_{1}, \ldots, b_{l}\right)$ are said to be disjoint if the subsets $\left\{a_{1}, \ldots, a_{k}\right\}$ and $\left\{b_{1}, \ldots, b_{l}\right\}$ are disjoint. It is well-known that every permutation is expressible as a composition of disjoint cycles (cf. [14, Theorem 5.1]).

Let $S$ be a semigroup. An element $a \in S$ is called an idempotent provided that $a^{2}=a$. The set of idempotents of $S$ is denoted by $E(S)$. It is well-known that $f \in \mathcal{T}_{X}$ is an idempotent if and only if $f$ acts as the identity map on its image set (cf. [5, pp. 6]). The group of units of $S$ is the subgroup of all invertible elements of $S$. An equivalence relation $\rho$ on $S$ is called a congruence if for all $x, y, z, w \in S),(x, y) \in \rho$ and $(z, w) \in \rho$ implies $(x z, y w) \in \rho$. If $\rho$ is a congruence on $S$, then the factor set $S / \rho$ is a semigroup, called a quotient semigroup, equipped with the multiplication defined by $[x][y]=[x y]$ for all $[x],[y] \in S / \rho$. We will write $S \cong T$ to mean that there is an isomorphism between two semigroups $S$ and $T$.

\section{The Semigroup $\Sigma(X, \mathcal{P})$}

In this section, we first characterize the elements of the semigroup $\Sigma(X, \mathcal{P})$. We then prove two simple but important lemmas on $S(X, \mathcal{P})$ and $\Sigma(X, \mathcal{P})$, respectively. We begin by recalling a definition.

Definition 3.1. (cf. [23]) Let $\mathcal{P}=\left\{X_{i} \mid i \in I\right\}$ be a partition of an arbitrary set $X$, and let $f \in T(X, \mathcal{P})$. The character of $f$ is a selfmap $\chi^{(f)}: I \rightarrow I$ defined by $i \chi^{(f)}=j$ whenever $X_{i} f \subseteq X_{j}$.

When $X$ is a finite set, the selfmap $\chi^{(f)}$ has been discussed, and also denoted by $\bar{f}$ in $[1,9,10$.

Denote by $S_{\mathcal{P}}(X)$ the semigroup of all continuous selfmaps on $X$ endowed with the topology having $\mathcal{P}$ as a basis. By [17, Theorem 2.8], we know that $S_{\mathcal{P}}(X)=$ $T(X, \mathcal{P})$. We now have the following. 
Theorem 3.2. Let $\mathcal{P}=\left\{X_{i} \mid i \in I\right\}$ be a partition of an arbitrary set $X$, and let $f \in T(X, \mathcal{P})$. Then the following statements are equivalent:

(i) $f \in \Sigma(X, \mathcal{P})$.

(ii) $\chi^{(f)}$ is a surjective map.

(iii) $f \in S_{\mathcal{P}}(X)$ such that $A f^{-1} \neq \emptyset$ for all nonempty open set $A$.

Proof. (i) $\Longrightarrow$ (ii). Let $j \in I$. Since $f \in \Sigma(X, \mathcal{P})$, we have $X f \cap X_{j} \neq \emptyset$. Then there exists $i \in I$ such that $X_{i} f \subseteq X_{j}$. It follows that $i \chi^{(f)}=j$ by definition of $\chi^{(f)}$. Hence $\chi^{(f)}$ is surjective.

(ii) $\Longrightarrow$ (iii). Let $A$ be a nonempty open set. Then $A=\bigcup_{j \in J} X_{j}$ for some $J \subseteq I$ (cf. 6, Definition 2.2.1]). Since $\chi^{(f)}: I \rightarrow I$ is surjective, for each $j \in J$ there exists $i \in I$ such that $i \chi^{(f)}=j$ and subsequently $X_{i} f \subseteq X_{j}$. That means $X_{j} f^{-1} \neq \emptyset$ for all $j \in J$. Hence we obtain

$$
A f^{-1}=\left(\bigcup_{j \in J} X_{j}\right) f^{-1}=\bigcup_{j \in J}\left(X_{j} f^{-1}\right) \neq \emptyset .
$$

(iii) $\Longrightarrow$ (i). Let $X_{i} \in \mathcal{P}$. By (iii), we have $X_{i} f^{-1} \neq \emptyset$. It follows that $X f \cap X_{i} \neq$ $\emptyset$ and hence $f \in \Sigma(X, \mathcal{P})$.

Definition 3.3 (cf. 20]). Let $E$ be an equivalence relation on a set $X$. A selfmap $f: X \rightarrow X$ is said to be $E^{*}$-preserving if $f$ satisfies the following.

$$
(x, y) \in E \text { if and only if }(x f, y f) \in E .
$$

We next have the following.

Theorem 3.4. Let $\mathcal{P}=\left\{X_{i} \mid i \in I\right\}$ be the partition associated with an equivalence relation $E$ on an arbitrary set $X$, and let $f \in T(X, \mathcal{P})$. Then the following statements are equivalent:

(i) $\chi^{(f)}$ is an injective map.

(ii) $f$ is an $E^{*}$-preserving map.

Proof. (i) $\Longrightarrow$ (ii). Let $x, y \in X$. Since $f \in T(X, \mathcal{P})$, it is clear that if $(x, y) \in E$ then $(x f, y f) \in E$. On the other hand, assume that $(x f, y f) \in E$. Then there exists $X_{l} \in \mathcal{P}$ such that $x f, y f \in X_{l}$. We now claim that $x, y \in X_{r}$ for some $X_{r} \in \mathcal{P}$.

On the contrary, suppose that $x \in X_{s}$ and $y \in X_{t}$ for two distinct blocks $X_{s}, X_{t} \in \mathcal{P}$. Since $x f, y f \in X_{l}$, and $f \in T(X, \mathcal{P})$, we have $X_{s} f \subseteq X_{l}$ and $X_{t} f \subseteq X_{l}$. Then $s \chi^{(f)}=l=t \chi^{(f)}$ by definition of $\chi^{(f)}$. This contradicts the hypothesis that $\chi^{(f)}$ is injective. Hence $x, y \in X_{r}$ for some $X_{r} \in \mathcal{P}$ and consequently $f$ is $E^{*}$ preserving.

(ii) $\Longrightarrow$ (i). By contradiction, suppose that there are two distinct elements $s, t \in I$ such that $s \chi^{(f)}=t \chi^{(f)}$, say equal to $r$. Then $X_{s} f \subseteq X_{r}$ and $X_{t} f \subseteq X_{r}$ by definition of $\chi^{(f)}$. Let $x \in X_{s}$ and $y \in X_{t}$. Since $X_{s} \cap X_{t}=\emptyset$, it is clear that $(x, y) \notin E$.

Recall that $X_{s} f \subseteq X_{r}$ and $x \in X_{s}$, it follows that $x f \in X_{r}$. Also $X_{t} f \subseteq X_{r}$ and $y \in X_{t}$, it follows that $y f \in X_{r}$. Thus $(x f, y f) \in E$ which contradicts the hypothesis that $f$ is an $E^{*}$-preserving map. Hence $\chi^{(f)}$ is an injective map. 
Note that any selfmap on a finite set that is injective or surjective must be bijective (cf. [15, Proposition 1.1.3]). Combining this fact with Theorem 3.2 and Theorem 3.4 we have the following direct corollary.

Corollary 3.5. Let $\mathcal{P}=\left\{X_{1}, \ldots, X_{m}\right\}$ be an m-partition associated with an equivalence relation $E$ on an arbitrary set $X$, and let $f \in T(X, \mathcal{P})$. Then the following four statements are equivalent:

(i) $f \in \Sigma(X, \mathcal{P})$.

(ii) $\chi^{(f)}$ is a bijective map on $I_{m}$.

(iii) $f$ is an $E^{*}$-preserving map.

(iv) $f \in S_{\mathcal{P}}(X)$ such that $A f^{-1} \neq \emptyset$ for all nonempty open set $A$.

Lemma 3.6. Let $\mathcal{P}=\left\{X_{i} \mid i \in I\right\}$ be a partition of an arbitrary set $X$, and let $f \in T(X, \mathcal{P})$. If $f \in S(X, \mathcal{P})$, then

(i) $X_{i} f \in \mathcal{P}$ for all $X_{i} \in \mathcal{P}$.

(ii) $\left|X_{i}\right|=\left|X_{j}\right|$ if $i \chi^{(f)}=j$.

Proof. Note that any two blocks of a partition are either equal or disjoint.

(i) On the contrary, suppose that $X_{i} f \notin \mathcal{P}$. Then there exists $X_{j} \in \mathcal{P}$ such that $X_{i} f \subsetneq X_{j}$. Since $f \in S(X, \mathcal{P})$ and $S(X, \mathcal{P})$ is a subgroup, it follows that the inverse map $f^{-1}$ of the bijection $f$ belongs to $S(X, \mathcal{P})$. Then $X_{j} f^{-1} \subseteq X_{k}$ for some $X_{k} \in \mathcal{P}$. Thus we obtain

$$
X_{i}=\left(X_{i} f\right) f^{-1} \subsetneq X_{j} f^{-1} \subseteq X_{k},
$$

which contradicts the fact that any two blocks are either equal or disjoint. Hence $X_{i} f \in \mathcal{P}$.

(ii) If $i \chi^{(f)}=j$, then $X_{i} f \subseteq X_{j}$ by definition of $\chi^{(f)}$. By (i), we have $X_{i} f \in \mathcal{P}$. Recall that any two blocks are either equal or disjoint. Since $X_{j} \in \mathcal{P}$, we have $X_{i} f=X_{j}$ whence $\left|X_{i}\right|=\left|X_{j}\right|$ since $f$ is a bijection.

We next recall the following congruence on the semigroup $T(X, \mathcal{P})$ which is induced by $\chi^{(f)}$.

Remark 3.7. (cf. 23]) Let $\mathcal{P}=\left\{X_{i} \mid i \in I\right\}$ be a partition of an arbitrary set $X$. Then the relation $\chi$ on $T(X, \mathcal{P})$ defined by

$$
(f, g) \in \chi \Longleftrightarrow \chi^{(f)}=\chi^{(g)}
$$

is a congruence on the semigroup $T(X, \mathcal{P})$.

Note that the congruence $\chi$ defined above on the semigroup $T(X, \mathcal{P})$ is also a congruence on the semigroup $\Sigma(X, \mathcal{P})$, and the quotient semigroup $\Sigma(X, \mathcal{P}) / \chi$ is a subsemigroup of $T(X, \mathcal{P}) / \chi$. We now prove the following proposition.

Lemma 3.8. Let $\mathcal{P}=\left\{X_{i} \mid i \in I\right\}$ be a partition of a set $X$. Then the semigroup $\Sigma(X, \mathcal{P}) / \chi$ is isomorphic to the semigroup of all surjective selfmaps on $I$.

Proof. Denote by $\mathcal{O}(I)$ the semigroup of all surjective selfmaps on $I$. Define a map $\phi: \Sigma(X, \mathcal{P}) / \chi \rightarrow \mathcal{O}(I)$ by setting $[f] \phi=\chi^{(f)}$ for all $[f] \in \Sigma(X, \mathcal{P}) / \chi$. By Theorem 3.2. it is clear that $\phi$ is well-defined. 
Let $[f],[g] \in \Sigma(X, \mathcal{P}) / \chi$. Note that $\chi^{(f g)}=\chi^{(f)} \chi^{(g)}$ (cf. [23, Lemma 2.3]. Therefore, we obtain

$$
([f][g]) \phi=\chi^{(f g)}=\chi^{(f)} \chi^{(g)}=([f] \phi)([g] \phi)
$$

whence $\phi$ is a homomorphism. To see $\phi$ is injective, suppose that $[f] \phi=[g] \phi$. Then

$$
\chi^{(f)}=\chi^{(g)} \Longrightarrow(f, g) \in \chi \Longrightarrow[f]=[g]
$$

and $\phi$ is injective.

To show that $\phi$ is surjective, let $\psi \in \mathcal{O}(I)$. For each $j \in I$, we arbitrarily choose an element $x_{j} \in X_{j}$. Define a map $f: X \rightarrow X$ by setting $x f=x_{j}$ whenever $x \in X_{i}$ and $i \psi=j$. Since $\psi$ is surjective, one can verify, in a routine manner, that $f \in \Sigma(X, \mathcal{P})$ and $\chi^{(f)}=\psi$. Therefore, $[f] \phi=\chi^{(f)}=\psi$ whence $\phi$ is surjective. This completes the proof.

\section{Permutations that Preserve Nontrivial Partition}

When $\mathcal{P}$ is a trivial partition of $X$, it is clear that $T(X, \mathcal{P})=\mathcal{T}_{X}$ and $S(X, \mathcal{P})=$ $\mathcal{S}_{X}$. Observe that $T(X, \mathcal{P}) \subsetneq \mathcal{T}_{X}$ and $S(X, \mathcal{P}) \subsetneq \mathcal{S}_{X}$ for all nontrivial partition $\mathcal{P}$ of $X$. We will now naturally be concerned of the converse problem: for a map $f \in \mathcal{T}_{X}$ $\left(f \in \mathcal{S}_{X}\right)$, does there exist a nontrivial partition $\mathcal{P}$ of $X$ such that $f \in T(X, \mathcal{P})$ $(f \in S(X, \mathcal{P}))$ ? This section deals with this interesting problem when $X$ is finite. In the rest of this section, let $X=\{1, \ldots, n\}$.

Let $f \in T_{n} \backslash S_{n}$. If $f$ is a constant map, it is clear that $f \in T(X, \mathcal{P})$ for each nontrivial partition $\mathcal{P}$ of $X$. Otherwise if $f$ is a nonconstant map, we see that $f \in T(X, \mathcal{P})$ where $\mathcal{P}$ is the nontrivial partition of $X$ induced by the equivalence relation $\operatorname{ker}(f)$ on $X$ defined as $\operatorname{ker}(f)=\{(x, y) \mid x f=y f\}$. Thus,

Remark 4.1. If $f \in T_{n} \backslash S_{n}$, then there exists a nontrivial partition $\mathcal{P}$ of $X$ such that $f \in T(X, \mathcal{P})$.

Proposition 4.2. Let $f \in S_{n}$. If $f$ is not an $n$-cycle, then there exists a nontrivial partition $\mathcal{P}$ of $X$ such that $f \in S(X, \mathcal{P})$.

Proof. The result is obvious for the identity permutation since the identity permutation preserves each partition of $X$. Therefore, suppose that $f$ is a nonidentity permutation. If $f$ is not an $n$-cycle, we can write

$$
f=\beta_{1} \beta_{2} \cdots \beta_{s}
$$

where $\beta_{i}$ 's are cycles of length less than $n$ (cf. 14, Theorem 5.1]). Let $X_{1}=\{x \in$ $\left.X \mid x \beta_{1} \neq x\right\}$. It is clear that $X_{1} f=X_{1}$ and $\left(X \backslash X_{1}\right) f=\left(X \backslash X_{1}\right)$. Moreover, one can verify, in a routine manner, that $\mathcal{P}=\left\{X_{1}, X \backslash X_{1}\right\}$ is a nontrivial partition of $X$. Hence, $f \in S(X, \mathcal{P})$ where $\mathcal{P}$ is a nontrivial partition of $X$.

Theorem 4.3. Let $f \in S_{n}$ be an $n$-cycle, and let $m$ be an integer such that $1<$ $m<n$. Then $m$ divides $n$ if and only if there exists a nontrivial $m$-partition $\mathcal{P}$ of $X$ such that $f \in S(X, \mathcal{P})$.

Proof. We first assume that $n=k m$ for some integer $k$ with $1<k<n$. Without loss of generality, let $f=(1, \ldots, n)$ be an $n$-cycle. Consider, for each $1 \leq i \leq m$, the subset

$$
X_{i}=\{i, i+m, i+2 m, \ldots, i+(k-1) m\} .
$$


of $X$. One can verify, in a routine manner, that $X_{i} \cap X_{j}=\emptyset$ for $i \neq j$, and $X=\bigcup_{i \in I_{m}} X_{i}$. This implies $\mathcal{P}=\left\{X_{1}, \ldots, X_{m}\right\}$ is a nontrivial $m$-partition of $X$. Since $f=(1,2, \ldots, n)$, we next see that $X_{i} f=X_{i+1}$ for $1 \leq i \leq m-1$ and $X_{m} f=X_{1}$. Hence $f \in S(X, \mathcal{P})$ where $\mathcal{P}$ is a nontrivial $m$-partition of $X$.

Conversely, assume that $\mathcal{P}=\left\{X_{1}, \ldots, X_{m}\right\}$ is a nontrivial $m$-partition of $X$ such that $f \in S(X, \mathcal{P})$. Since $f \in S(X, \mathcal{P})$, by Lemma 3.6(i), we have $X_{i} f \in \mathcal{P}$ for all $i \in I_{m}$. We first claim that $X_{i} f \neq X_{i}$ for all $i \in I_{m}$. If possible, let $X_{s} f=X_{s}$ for some $s \in I_{m}$. Then $\left(X \backslash X_{s}\right) f=X \backslash X_{s}$. It follows that that $f$ is not an $n$-cycle, which is a contradiction. Hence $X_{i} f \neq X_{i}$ for all $i \in I_{m}$.

Let $i, j \in I_{m}$ such that $i \neq j$. If $X_{i} f=X_{j}$, then we claim that $X_{j} f \neq X_{i}$. If possible, let $X_{j} f=X_{i}$. Then $f$ is not an $n$-cycle, which is a contradiction. Thus, without loss of generality, assume that $X_{i} f=X_{i+1}$ for each $1 \leq i \leq m-1$ and $X_{m} f=X_{1}$. Since $f \in S(X, \mathcal{P})$, by Lemma 3.6(ii), we have $\left|X_{1}\right|=\cdots=\left|X_{m}\right|$. Then letting $\left|X_{i}\right|=t$ for each $i \in I_{m}$, we obtain

$$
\left|X_{1}\right|+\cdots+\left|X_{m}\right|=n \Longrightarrow t m=n
$$

whence $m$ divides $n$. This completes the proof.

The following corollary is an immediate consequence of Theorem 4.3 .

Corollary 4.4. Let $f \in S_{n}$ be an $n$-cycle. If $n$ is a prime, then $f \notin S(X, \mathcal{P})$ for any nontrivial partition $\mathcal{P}$ of $X$.

Proposition 4.5. Let $\mathcal{P}=\left\{X_{1}, \ldots, X_{m}\right\}$ be an m-partition of $X$, and let $f \in$ $S(X, \mathcal{P})$. If $f$ is an $n$-cycle, then

(i) $\chi^{(f)}$ is an $m$-cycle on $I_{m}$.

(ii) $\mathcal{P}$ is a uniform partition of $X$.

Proof.

(i) Note that $S(X, \mathcal{P}) \subseteq \Sigma(X, \mathcal{P})$. In view of Corollary 3.5, $\chi^{(f)}$ is a permutation of $I_{m}$. If $f$ is an $n$-cycle, then $m$ divides $n$ by Theorem 4.3, If $m$ is an improper divisor of $n$, one can easily verify, in a routine manner, that $\chi^{(f)}$ is an $m$-cycle. Otherwise, assume that $m$ is a proper divisor of $n$.

On the contrary, suppose that $\chi^{(f)}$ is not an $m$-cycle. Then $\chi^{(f)}$ can be written as a product of disjoint cycles (cf. [14, Theorem 5.1]). Let $\left(i_{1}, \ldots, i_{t}\right)$ be a $t$-cycle, $1<t<m$, in the cycle decomposition of $\chi^{(f)}$. Then $i_{r} \chi^{(f)}=i_{r+1}$ for $r=1, \ldots, t-1$ and $i_{t} \chi^{(f)}=i_{1}$.

Since $f \in S(X, \mathcal{P})$, by Lemma $\underline{3.6}$ (i) we thus obtain $X_{i_{r}} f=X_{i_{r+1}}$ for all $r=1, \ldots, t-1$ and $X_{i_{t}} f=X_{i_{1}}$. This means that $f$ is a cycle of length at most $t k$, which is obviously less than $n$, where $k=\left|X_{i_{j}}\right|$ for each $j=1, \ldots, t$. This gives a contradiction of the hypothesis that $f$ is an $n$-cycle. Hence $\chi^{(f)}$ is an $m$-cycle on $I_{m}$.

(ii) Without loss of generality, assume that $\chi^{(f)}=(1, \ldots, m)$ by (i). This means $i \chi^{(f)}=i+1$ for all $i=1, \ldots, m-1$ and $m \chi^{(f)}=1$. Since $f \in S(X, \mathcal{P})$, by Lemma 3.6(ii) we have $\left|X_{1}\right|=\cdots=\left|X_{m}\right|$ whence $\mathcal{P}$ is a uniform partition of $X$. 


\section{IDEMPotents in $\Sigma(X, \mathcal{P})$}

In this section, we first define a notion of block maps associated with a set partition. We then proceed with lemmas and a corollary. By using these lemmas, we next characterize the idempotents of the semigroup $\Sigma(X, \mathcal{P})$. We also prove a necessary and sufficient condition for a map of $T(X, \mathcal{P})$ that belongs to $S(X, \mathcal{P})$. We finally count the idempotents in the semigroup $\Sigma(X, \mathcal{P})$ when $X$ is finite.

Definition 5.1. Let $\mathcal{P}$ be a partition of an arbitrary set. A block map is a map whose both domain and codomain are blocks of $\mathcal{P}$.

We now prove the following simple but important lemmas.

Lemma 5.2. Let $\mathcal{P}=\left\{X_{i} \mid i \in I\right\}$ be a partition of a set $X$, and let $f \in \mathcal{T}_{X}$. Then $f \in T(X, \mathcal{P})$ if and only if there exists a unique indexed family $B(f, I)$ of block maps induced by $f$, where

$$
B(f, I)=\left\{f_{i} \mid f_{i} \text { is induced by fand } \operatorname{dom}\left(f_{i}\right)=X_{i} \text { for each } i \in I\right\} .
$$

Proof. We first assume that $f \in T(X, \mathcal{P})$, and let $X_{i} \in \mathcal{P}$. Then there exists $X_{j} \in \mathcal{P}$ such that $X_{i} f \subseteq X_{j}$. We subsequently have a block map from $X_{i}$ to $X_{j}$ induced by $f$. Denote this induced block map by $f_{i}$. Since $X_{i} \in \mathcal{P}$ is an arbitrary block, we therefore obtain a unique indexed family $B(f, I)$ of block maps induced by $f$, where $B(f, I)=\left\{f_{i} \mid f_{i}\right.$ is induced by $f$ and $\operatorname{dom}\left(f_{i}\right)=X_{i}$ for each $\left.i \in I\right\}$.

Conversely, suppose that the condition holds. Let $X_{i} \in \mathcal{P}$. Then $X_{i} f=X_{i} f_{i}$. Since $\operatorname{codom}\left(f_{i}\right) \in \mathcal{P}$, we have $X_{i} f \subseteq X_{j}$, where $X_{j}=\operatorname{Codom}\left(f_{i}\right)$. Hence $f \in$ $T(X, \mathcal{P})$. This completes the proof.

Lemma 5.3. Let $\mathcal{P}=\left\{X_{i} \mid i \in I\right\}$ be a partition of a set $X$, and let $f \in T(X, \mathcal{P})$. If each block map of $B(f, I)$ is an idempotent, then $f$ is an idempotent.

Proof. Let $x \in X$. Then $x \in X_{i}$ for some $i \in I$. Since $f_{i} \in B(f, I)$ is an idempotent, it follows that $f_{i}: X_{i} \rightarrow X_{i}$. Therefore

$$
x\left(f^{2}\right)=(x f) f=\left(x f_{i}\right) f=\left(x f_{i}\right) f_{i}=x\left(f_{i}^{2}\right)=x f_{i}=x f
$$

whence $f$ is an idempotent.

Lemma 5.4. Let $\mathcal{P}=\left\{X_{i} \mid i \in I\right\}$ be a partition of a set $X$, and let $f \in$ $E(T(X, \mathcal{P}))$. If $i \in I \chi^{(f)}$, then the block map $f_{i} \in B(f, I)$ is an idempotent.

Proof. If $i \in I \chi^{(f)}$, then we see that $X f \cap X_{i} \neq \emptyset$. Let $y \in X f \cap X_{i}$. Since $f \in E\left(T(X, \mathcal{P})\right.$ ), we have $y f=y$ (cf. [5] pp. 6]). If follows that $f_{i}: X_{i} \rightarrow X_{i}$. Let $x \in X_{i}$. Then

$$
x\left(f_{i}^{2}\right)=\left(x f_{i}\right) f_{i}=(x f) f_{i}=x\left(f^{2}\right)=x f=x f_{i}
$$

whence the block map $f_{i}$ is an idempotent.

Corollary 5.5. Let $\mathcal{P}=\left\{X_{i} \mid i \in I\right\}$ be a partition of a set $X$, and let $f \in T(X, \mathcal{P})$. If $f$ is an idempotent, then $\chi^{(f)}$ is an idempotent.

Proof. Let $j \in I \chi^{(f)}$. It is sufficient to show that $j \chi^{(f)}=j$ (cf. [5, pp. 6]). Since $j \in I \chi^{(f)}$, the block map $f_{j} \in B(f, I)$ is an idempotent by Lemma 5.4. It follows that $f_{j}: X_{j} \rightarrow X_{j}$ and subsequently $j \chi^{(f)}=j$. Hence $\chi^{(f)}$ is an idempotent. 
The following theorem which characterizes the idempotents of the semigroup $\Sigma(X, \mathcal{P})$ is a direct consequence of Lemma 5.3 and Lemma 5.4

Theorem 5.6. Let $\mathcal{P}=\left\{X_{i} \mid i \in I\right\}$ be a partition of a set $X$, and let $f \in \Sigma(X, \mathcal{P})$. Then $f$ is an idempotent if and only if each block map of $B(f, I)$ is an idempotent.

Theorem 5.6 yields the following.

Corollary 5.7. Let $\mathcal{P}=\left\{X_{i} \mid i \in I\right\}$ be a partition of a set $X$, and let $f \in \Sigma(X, \mathcal{P})$. If $f$ is an idempotent, then $\chi^{(f)}$ is the identity map.

Proof. Let $i \in I$. If $f \in \Sigma(X, \mathcal{P})$ is an idempotent, then $f_{i}: X_{i} \rightarrow X_{i}$ by Theorem 5.6. It follows that $X_{i} f=X_{i} f_{i} \subseteq X_{i}$. By definition of $\chi^{(f)}$, we then have $i \chi^{(f)}=i$. Hence $\chi^{(f)}$ is the identity map on $I$.

The next theorem provides a necessary and sufficient condition for a map of $T(X, \mathcal{P})$ that belongs to $S(X, \mathcal{P})$.

Theorem 5.8. Let $\mathcal{P}=\left\{X_{i} \mid i \in I\right\}$ be a partition of an arbitrary set $X$, and let $f \in T(X, \mathcal{P})$. Then $f \in S(X, \mathcal{P})$ if and only if

(i) each block map of $B(f, I)$ is a bijective map; and

(ii) $\chi^{(f)}$ is a bijective map.

Proof. Assume that $f \in S(X, \mathcal{P})$. Let $f_{i} \in B(f, I)$. It is clear that the block map $f_{i}$ is injective. If $\operatorname{Codom}\left(f_{i}\right)=X_{j}$, then $X_{i} f=X_{i} f_{i} \subseteq X_{j}$. Since $f \in S(X, \mathcal{P})$, we have $X_{i} f_{i} \in \mathcal{P}$ by Lemma 3.6(i). We then have $X_{i} f_{i}=X_{j}$ and so $f_{i}$ is surjective. Since $f_{i} \in B(f, I)$ is arbitrary, this proves (i).

We next prove (ii). Note that $S(X, \mathcal{P}) \subseteq \Sigma(X, \mathcal{P})$. Since $f \in S(X, \mathcal{P})$, by Theorem 3.2. the map $\chi^{(f)}$ is surjective. On the contrary, suppose that there exist two distinct elements $i, j \in I$ such that $i \chi^{(f)}=j \chi^{(f)}$, say equal to $k$ for some $k \in I$. Then $X_{i} f \subseteq X_{k}$ and $X_{j} f \subseteq X_{k}$ by definition of $\chi^{(f)}$. Since $f \in S(X, \mathcal{P})$, by Lemma 3.6(i), we have $X_{i} f=X_{k}$ and $X_{j} f=X_{k}$ and subsequently $X_{i}=X_{j}$. This is a contradiction since $i \neq j$. Hence the map $\chi^{(f)}$ is bijective.

Conversely, suppose that the conditions hold. It is sufficient to prove that $f$ is a bijection. We first prove that $f$ is injective. Let $x, y \in X$ and suppose that $x f=y f$. Then $x f, y f \in X_{j}$ for some $j \in I$. By (ii), there exists $i \in I$ such that $x, y \in X_{i}$. By (i), we know that $f_{i}$ is injective. We thus obtain $x f=y f \Longrightarrow x f_{i}=$ $y f_{i} \Longrightarrow x=y$. Therefore $f$ is injective.

We now prove that $f$ is surjective. Let $y \in X$. Then $y \in X_{j}$ for some $j \in I$. By (ii), there exists $i \in I$ such that $i \chi^{(f)}=j$ and so $X_{i} f \subseteq X_{j}$. It follows that $f_{i}: X_{i} \rightarrow X_{j}$. By (i), there exists $x \in X_{i}$ such that $x f_{i}=y$ and subsequently $x f=y$. Therefore $f$ is surjective. This completes the proof.

When $X$ is a finite set, the following theorem counts the number of the idempotents in the semigroup $\Sigma(X, \mathcal{P})$.

Theorem 5.9. Let $\mathcal{P}=\left\{X_{1}, \ldots, X_{m}\right\}$ be an $(m, k)$-partition of a finite set $X$ such that $\mathcal{P}$ has exactly $m_{i} \geq 1$ blocks of size $n_{i} \geq 1$ for each $i \in I_{k}$. Then

$$
|E(\Sigma(X, \mathcal{P}))|=\prod_{i=1}^{k}\left(\sum_{j=1}^{n_{i}}\left(\begin{array}{c}
n_{i} \\
j
\end{array}\right) j^{n_{i}-j}\right)^{m_{i}} .
$$


Proof. Note that each map $f \in \Sigma(X, \mathcal{P})$ is uniquely determined by the $m$-family $B\left(f, I_{m}\right)$ of block maps (cf. Lemma 5.2). From Theorem 5.6, we know that a map $f \in \Sigma(X, \mathcal{P})$ is an idempotent if and only if each block map $f_{i} \in B\left(f, I_{m}\right)$ is an idempotent. It is therefore sufficient to count the total number of such $m$-families $B\left(f, I_{m}\right)$ of idempotent block maps. To count it, we break up the problem into $k$ subfamilies depending on the domain sizes of block maps.

Let $i \in I_{k}$. Since $\mathcal{P}$ has $m_{i}$ blocks of size $n_{i}$, we begin by counting the number of possible $m_{i}$-subfamilies of idempotent block maps from $m_{i}$ distinct blocks of size $n_{i}$. Note that the total number of idempotents in the full transformation semigroup on an $n$-element set is $\sum_{j=1}^{n}\left(\begin{array}{c}n \\ j\end{array}\right) j^{n-j}$ (cf. [15, Corollary 2.7.4]). Moreover, any map which is an idempotent must be selfmap. Therefore, the number of possible idempotent block maps from a block of size $n_{i}$ is $\sum_{j=1}^{n_{i}}\left(\begin{array}{c}n_{i} \\ j\end{array}\right) j^{n_{i}-j}$.

Recall that $\mathcal{P}$ has $m_{i}$ blocks of size $n_{i}$, by the multiplication principle, the total number of possible $m_{i}$-subfamilies of idempotent block maps from $m_{i}$ distinct blocks of size $n_{i}$ is $\left(\sum_{j=1}^{n_{i}}\left(\begin{array}{c}n_{i} \\ j\end{array}\right) j^{n_{i}-j}\right)^{m_{i}}$. Since $\mathcal{P}$ has exactly $k$ different size blocks and $i \in I_{k}$ is arbitrarily choosen element, the total number of possible $m$-families of idempotent block maps is now follows by applying the multiplication principle. This completes the proof.

\section{The cardinality of $T(X, \mathcal{P}), \Sigma(X, \mathcal{P})$, and $S(X, \mathcal{P})$}

This section calculates the size of $T(X, \mathcal{P}), S(X, \mathcal{P})$, and $\Sigma(X, \mathcal{P})$, respectively when $X$ is a finite set. We begin by calculating the cardinality of the semigroup $T(X, \mathcal{P})$.

Theorem 6.1. Let $\mathcal{P}=\left\{X_{1}, \ldots, X_{m}\right\}$ be an $(m, k)$-partition of a finite set $X$ such that $\mathcal{P}$ has exactly $m_{i} \geq 1$ blocks of size $n_{i} \geq 1$ for each $i \in I_{k}$. Then

$$
|T(X, \mathcal{P})|=\prod_{i=1}^{k}\left(\sum_{j=1}^{k} m_{j} n_{j}^{n_{i}}\right)^{m_{i}} .
$$

Proof. Note that each map $f \in T(X, \mathcal{P})$ is uniquely determined by the $m$-family $B\left(f, I_{m}\right)$ of block maps (cf. Lemma 5.2). Therefore, it is sufficient to count the total number of such $m$-families $B\left(f, I_{m}\right)$ of block maps. To count it, we break up the problem into $k$ subfamilies depending on the domain sizes of block maps.

Let $i \in I_{k}$. Since $\mathcal{P}$ has $m_{i}$ blocks of size $n_{i}$, we begin by counting the number of possible $m_{i}$-subfamilies of block maps from $m_{i}$ distinct blocks of size $n_{i}$. Clearly, the codomain of a block map from a block of size $n_{i}$ can be any block of $\mathcal{P}$. Note that the number of maps from an $n$-element set into an $t$-element set is $t^{n}$. Therefore, the number of possible block maps from a block of size $n_{i}$ is $\sum_{j=1}^{k} m_{j} n_{j}^{n_{i}}$ by the addition principle.

Recall that $\mathcal{P}$ has $m_{i}$ blocks of size $n_{i}$, by the multiplication principle, the total number of possible $m_{i}$-subfamilies of block maps from $m_{i}$ distinct blocks of size $n_{i}$ is $\left(\sum_{j=1}^{k} m_{j} n_{j}^{n_{i}}\right)^{m_{i}}$. Since $\mathcal{P}$ has exactly $k$ different size blocks and $i \in I_{k}$ is arbitrarily choosen element, the total number of possible $m$-families of block maps is now follows by applying the multiplication principle. This completes the proof. 
The next theorem calculate the cardinality of the group of units $S(X, \mathcal{P})$ of the semigroup $T(X, \mathcal{P})$.

Theorem 6.2. Let $\mathcal{P}=\left\{X_{1}, \ldots, X_{m}\right\}$ be an $(m, k)$-partition of a finite set $X$ such that $\mathcal{P}$ has exactly $m_{i} \geq 1$ blocks of size $n_{i} \geq 1$ for each $i \in I_{k}$. Then

$$
|S(X, \mathcal{P})|=\prod_{i=1}^{k}\left(m_{i} !\right)\left(n_{i} !\right)^{m_{i}}
$$

Proof. Consider the equivalence relation $\sim$ on the partition $\mathcal{P}$ defined by $P \sim Q$ if and only if $|P|=|Q|$. Clearly, there are exactly $k$ equivalence classes under the equivalence $\sim$. Let $\left[X_{1}\right], \ldots,\left[X_{k}\right]$ be the equivalence classes under $\sim$, where $\left|X_{i}\right|=n_{i}$. Note that $\left|\left[X_{i}\right]\right|=m_{i}$ for each $i=1, \ldots, k$.

Let $i \in I_{k}$. Consider the class $\left[X_{i}\right]$. By Lemma 3.6 and Theorem $[5.8($ ii), we first note that the images of two distinct blocks in $\left[X_{i}\right]$ under a map of $S(X, \mathcal{P})$ must be distinct blocks in $\left[X_{i}\right]$. If $f \in S(X, \mathcal{P})$, then there are $m_{i}$ choices for the image of the first block of $\left[X_{i}\right]$ under $f$, the remaining $\left(m_{i}-1\right)$ choices for the image of the second block of $\left[X_{i}\right]$ under $f$, etc. For the last block of $\left[X_{i}\right]$, there is exactly one choice under $f$. Therefore, by the multiplication principle, all $m_{i}$ blocks of the class $\left[X_{i}\right]$ can be mapped in $m_{i}$ ! different ways.

Note that the number of bijections between any two $n$-element sets is $n !$. Therefore, among $m_{i}$ ! different choices, each fixed choice gives $\left(n_{i} !\right)^{m_{i}}$ bijections that preserve all blocks of $\left[X_{i}\right]$ by the multiplication principle. Hence, the total number of bijections that preserve all blocks of the class $\left[X_{i}\right]$ is $\left(m_{i} !\right)\left(n_{i} !\right)^{m_{i}}$.

Since there are exactly $k$ equivalence classes and $\left[X_{i}\right]$ is arbitrarily choosen class, one can obtain the stated formula of $|S(X, \mathcal{P})|$ by the multiplication principle.

The following theorem calculate the cardinality of the semigroup $\Sigma(X, \mathcal{P})$.

Theorem 6.3. Let $\mathcal{P}=\left\{X_{1}, \ldots, X_{m}\right\}$ be an $(m, k)$-partition of a finite set $X$ such that $\mathcal{P}$ has exactly $m_{i} \geq 1$ blocks of size $n_{i} \geq 1$ for each $i \in I_{k}$. Then

$$
|\Sigma(X, \mathcal{P})|=m_{1} ! \ldots m_{k} ! \sum n_{1}^{s\left(m_{1}\right)} \ldots n_{k}^{s\left(m_{k}\right)}
$$

where the sum runs over all $k$-tuple of

$$
A:=\left\{\left(t_{m_{1}}, \ldots, t_{m_{k}}\right) \mid \forall i \in I_{k}, t_{m_{i}}=\left(l_{1}, \ldots, l_{m_{i}}\right), l_{j} \in\left\{n_{1}, \ldots, n_{k}\right\}\right\}
$$

such that the components of all $t_{m_{i}}$ 's of a $k$-tuple of $A$ form $\left\{m_{1} \cdot n_{1}, \ldots, m_{k} \cdot n_{k}\right\}$; each $s\left(m_{i}\right)$ is the sum of all components of $t_{m_{i}}$ in a $k$-tuple of $A$.

Proof. Since $\mathcal{P}$ is an $m$-partition of a finite set $X$, by Lemma 3.8, we must have $\Sigma(X, \mathcal{P}) / \chi \cong S_{m}$. It follows that there are $m$ ! distinct equivalence classes into which $\Sigma(X, \mathcal{P})$ splits under the equivalence $\chi$. Therefore, it is sufficient to calculate the cardinality of all $m$ ! distinct equivalence classes under the equivalence $\chi$.

Let $[f] \in \Sigma(X, \mathcal{P}) / \chi$ be an arbitrary class. We now calculate the cardinality of the class $[f]$. By Lemma 5.2, it is sufficient to count the total number of $m$-families of block maps induced by maps of $[f]$. Note, for any $g, h \in[f]$, that $\chi^{(g)}=\chi^{(h)}$. From Corollary 3.5, we recall that the map $\chi^{(f)}$ is a bijection on $I_{m}$.

Let $X_{i} \in \mathcal{P}$. If $i \chi^{(f)}=i^{\prime}$, then $X_{i} f_{i}=X_{i} f \subseteq X_{i^{\prime}}$. Therefore the number of block maps from $X_{i}$ to $X_{i^{\prime}}$ is $r_{i^{\prime}}^{r_{i}}$, where $r_{i}, r_{i^{\prime}} \in\left\{m_{1} \cdot n_{1}, \ldots, m_{k} \cdot n_{k}\right\}$ and $\left|X_{i}\right|=$ 
$r_{i},\left|X_{i^{\prime}}\right|=r_{i^{\prime}}$. Since $\mathcal{P}$ has exactly $m$ distinct blocks, by the multiplication principle, the total number of possible $m$-families $B\left(f, I_{m}\right)$ of block maps is $r_{1^{\prime}}^{r_{1}} r_{2^{\prime}}^{r_{2}} \ldots r_{m^{\prime}}^{r_{m}}$. Thus $|[f]|=r_{1^{\prime}}^{r_{1}} r_{2^{\prime}}^{r_{2}} \ldots r_{m^{\prime}}^{r_{m}}$, where $r_{i}, r_{i^{\prime}} \in\left\{m_{1} \cdot n_{1}, \ldots, m_{k} \cdot n_{k}\right\}$ for all $1 \leq i, i^{\prime} \leq m$.

Since $[f] \in \Sigma(X, \mathcal{P}) / \chi$ is an arbitrary class, by the addition principle, we thus obtain

$$
|\Sigma(X, \mathcal{P})|=\sum_{[f] \in \Sigma(X, \mathcal{P}) / \chi}|[f]|=\sum_{\phi \in S_{m}} r_{1^{\prime}}^{r_{1}} r_{2^{\prime}}^{r_{2}} \ldots r_{m^{\prime}}^{r_{m}}
$$

where $\phi \in S_{m}$ denotes the isomorphic image of the class $[f]$ and $i \phi=i^{\prime}$. Since $\mathcal{P}$ has exactly $m_{i}$ blocks of size $n_{i}$ for each $i \in I_{k}$, we see that all the $r_{i}$ 's form the multiset $\left\{m_{1} \cdot n_{1}, \ldots, m_{k} \cdot n_{k}\right\}$, and also all $r_{i^{\prime}}$ 's form the multiset $\left\{m_{1} \cdot n_{1}, \ldots, m_{k} \cdot n_{k}\right\}$.

Hence, by [4, Theorem 2.4.2], we obtain

$$
|\Sigma(X, \mathcal{P})|=m_{1} ! \ldots m_{k} ! \sum n_{1}^{s\left(m_{1}\right)} \ldots n_{k}^{s\left(m_{k}\right)}
$$

where the sum runs over all $k$-tuple of

$$
A:=\left\{\left(t_{m_{1}}, \ldots, t_{m_{k}}\right) \mid \forall i \in I_{k}, t_{m_{i}}=\left(l_{1}, \ldots, l_{m_{i}}\right), l_{j} \in\left\{n_{1}, \ldots, n_{k}\right\}\right\}
$$

such that the components of all $t_{m_{i}}$ 's of a $k$-tuple of $A$ form $\left\{m_{1} \cdot n_{1}, \ldots, m_{k} \cdot n_{k}\right\}$; each $s\left(m_{i}\right)$ is the sum of all components of $t_{m_{i}}$ in a $k$-tuple of $A$.

\section{ACKNOWLEDGMENT}

The authors would like to thank the anonymous referee who provided critical and detailed comments on part of an earlier version of the manuscript.

\section{REFERENCES}

[1] J. Araújo, W. Bentz, J. D. Mitchell, and C. Schneider. The rank of the semigroup of transformations stabilising a partition of a finite set. Mathematical Proceedings of the Cambridge Philosophical Society, 159(2): 339-353, 2015.

[2] J. Araújo and J. Konieczny. Semigroups of transformations preserving an equivalence relation and a cross-section. Communications in Algebra, 32(5): 1917-1935, 2004.

[3] J. Araújo and C. Schneider. The rank of the endomorphism monoid of a uniform partition. Semigroup Forum, 78(3): 498-510, 2009.

[4] R. A. Brualdi. Introductory Combinatorics, 5th edition. Pearson Prentice Hall, 2010.

[5] A. H. Clifford and G. B. Preston. The Algebraic Theory of Semigroups, Volume I. American Mathematical Society, Number 7 in Mathematical Surveys, 1961.

[6] J. B. Conway. A Course in Point Set Topology. Undergraduate Texts in Mathematics, Springer, 2014.

[7] L. Z. Deng, J. W. Zeng, and T. J. You. Green's relations and regularity for semigroups of transformations that preserve reverse direction equivalence. Semigroup Forum, 83(3): 489498, 2011.

[8] L. Z. Deng, J. W Zeng, and T. J. You. Green's relations and regularity for semigroups of transformations that preserve order and a double direction equivalence. Semigroup Forum, 84(1): 59-68, 2012.

[9] I. Dolinka and J. East. Idempotent generation in the endomorphism monoid of a uniform partition. Communications in Algebra, 44(12): 5179-5198, 2016.

[10] I. Dolinka, J. East, and J. D. Mitchell. Idempotent rank in the endomorphism monoid of a non-uniform partition. Bulletin of the Australian Mathematical Society, 93(1): 73-91, 2016.

[11] V. H. Fernandes and T. M. Quinteiro. On the monoids of transformations that preserve the order and a uniform partition. Communications in Algebra, 39(8): 2798-2815, 2011. 
[12] V. H. Fernandes and T. M. Quinteiro. The cardinal of various monoids of transformations that preserve a uniform partition. Bulletin of the Malaysian Mathematical Sciences Society, 35(4): 885-896, 2012.

[13] V. H. Fernandes and T. M. Quinteiro. On the ranks of certain monoids of transformations that preserve a uniform partition. Communications in Algebra, 42(2): 615-636, 2014.

[14] J. A. Gallian. Contemporary Abstract Algebra, 9th edition. Cengage Learning, 2017.

[15] O. Ganyushkin and V. Mazorchuk. Classical Finite Transformation Semigroups, An Introduction. volume 9 of Algebra and Applications, Springer, 2009.

[16] J. M. Howie. Fundamentals of Semigroup Theory. Clarendon Press, 1995.

[17] H. Pei. Equivalences, $\alpha$-semigroups and $\alpha$-congruences. Semigroup Forum, 49(1): 49-58, 1994.

[18] H. Pei. Some $\alpha$-semigroups inducing certain lattices. Semigroup Forum, 57: 48-59, 1998.

[19] H. Pei. On the rank of the semigroup $T_{E}(X)$. Semigroup Forum, 70(1): 107-117, 2005.

[20] H Pei. Regularity and Green's relations for semigroups of transformations that preserve an equivalence. Communications in Algebra, 33(1): 109-118, 2005.

[21] P. Huisheng and D. Zou. Green's equivalences on semigroups of transformations preserving order and an equivalence relation. Semigroup Forum, 71(2): 241-251, 2005.

[22] H. Pei, L. Sun, and H. Zhai. Green's relations for the variants of transformation semigroups preserving an equivalence relation. Communications in Algebra, 35(6): 1971-1986, 2007.

[23] P. Purisang and J. Rakbud. Regularity of transformation semigroups defined by a partition. Communications of the Korean Mathematical Society, 31(2): 217-227, 2016.

[24] L. Sun. Combinatorial results for certain semigroups of transformations preserving orientation and a uniform partition. Bulletin of the Malaysian Mathematical Sciences Society, 36(1): 179-192, 2013.

[25] L. Sun, H. Pei, and Z. Cheng. Regularity and Green's Relations for Semigroups of Transformations Preserving Orientation and an Equivalence. Semigroup Forum, 74(3): 473-486, 2007.

[26] L. Sun and J. Sun. A partial order on transformation semigroups that preserve double direction equivalence relation. Journal of Algebra and Its Applications, 12(8): 1350041, 2013.

Department of Mathematics, Central University of South Bihar, Gaya,Bihar, India E-mail address: mosarofsarkar@cusb.ac.in

Department of Mathematics, Central University of South Bihar, Gaya, Bihar, India

E-mail address: shubh@cub.ac.in 